\title{
Weyl Symmetry and the Liouville Theory*ił
}

\author{
R. Jackiw \\ Center for Theoretical Physics \\ Department of Physics \\ Massachusetts Institute of Technology \\ Cambridge, MA 02139
}

MIT-CTP-3699

\begin{abstract}
Flat-space conformal invariance and curved-space Weyl invariance are simply related in dimensions greater than two. In two dimensions the Liouville theory presents an exceptional situation, which we here examine.
\end{abstract}

\footnotetext{
* Schnitzerfest, Waltham MA, March 2005

${ }^{\dagger}$ Polyakovfest, Princeton NJ, November 2005

${ }^{\ddagger}$ Novozhilov Festschrift, (Theoretical Mathematical Physics)
} 


\section{Conformally and Weyl Invariant Scalar Field Dynamics in} $d>2$

Let us begin by recording the d-dimensional Lagrange density for a scalar field $\varphi$ with a scale and conformally invariant self interaction.

$$
\mathcal{L}_{0}=\frac{1}{2} \eta^{\mu \nu} \partial_{\mu} \varphi \partial_{\nu} \varphi-\lambda \varphi^{\frac{2 d}{d-2}}
$$

Evidently the expression makes sense only for $d \neq 2$, and we take $d>2$. The theory is invariant against

$$
\delta \varphi=f^{\alpha} \partial_{\alpha} \varphi+\frac{d-2}{2 d} \partial_{\alpha} f^{\alpha} \varphi
$$

where $f^{\alpha}$ is a (flat-space) conformal Killing vector. The usual, canonical energy momentum tensor

$$
\theta_{\mu \nu}^{\text {canonical }}=\partial_{\mu} \varphi \partial_{\nu} \varphi-\eta_{\mu \nu}\left(\frac{1}{2} \eta^{\alpha \beta} \partial_{\alpha} \varphi \partial_{\beta} \varphi-\lambda \varphi^{\frac{2 d}{d-2}}\right)
$$

is conserved and symmetric, as it should be in a Poincaré invariant theory. But it is not traceless: $\eta^{\mu \nu} \theta_{\mu \nu}^{\text {canonical }} \neq 0$. Nevertheless, because of the conformal invariance (1.2), $\theta_{\mu \nu}^{\text {canonical }}$ can be improved by the addition of a further conserved and symmetric expression, so that the new tensor is traceless [1].

$$
\theta_{\mu \nu}=\theta_{\mu \nu}^{\text {canonical }}+\frac{d-2}{4(d-1)}\left(\eta_{\mu \nu} \square-\partial_{\mu} \partial_{\nu}\right) \varphi^{2}, \eta^{\mu \nu} \theta_{\mu \nu}=0
$$

A variational derivation of the canonical tensor (1.3) becomes possible after the theory (1.1) is minimally coupled to a metric tensor $g_{\mu \nu}$, and its action integral is varied with respect to $g^{\mu \nu}$. $\theta_{\mu \nu}^{\text {canonical }}$ is regained in the limit $g_{\mu \nu} \rightarrow \eta_{\mu \nu}$. A similar derivation of the improved tensor (1.4) is also possible, provided (1.1), generalized to curved space, is extended by a specific non-minimal coupling [1].

$$
\begin{gathered}
\mathcal{L}=\frac{d-2}{8(d-1)} R \varphi^{2}+\frac{1}{2} g^{\mu \nu} \partial_{\mu} \varphi \partial_{\nu} \varphi-\lambda \varphi^{\frac{2 d}{d-2}} \\
T_{\mu \nu}=\frac{2}{\sqrt{|g|}} \frac{\delta}{\delta g^{\mu \nu}} \int \sqrt{|g|} \mathcal{L} \\
=\partial_{\mu} \varphi \partial_{\nu} \varphi-g_{\mu \nu}\left(\frac{1}{2} g^{\alpha \beta} \partial_{\alpha} \varphi \partial_{\beta} \varphi-\lambda \varphi^{\frac{2 d}{d-2}}\right)+\frac{d-2}{4(d-1)}\left(g_{\mu \nu} D^{2}-D_{\mu} D_{\nu}+G_{\mu \nu}\right) \varphi^{2}
\end{gathered}
$$

Here $G_{\mu \nu}$ is the Einstein tensor, $R$ the Ricci scalar $R=\frac{2}{2-d} g^{\mu \nu} G_{\mu \nu}$, and $D_{\mu}$ the covariant derivative. In the limit $g_{\mu \nu} \rightarrow \eta_{\mu \nu}$ the non-minimal term in $\mathcal{L}$ vanishes, but it survives in the $g^{\mu \nu}$ variation.

$$
T_{\mu \nu} \underset{g_{\mu \nu} \rightarrow \eta_{\mu \nu}}{\longrightarrow} \theta_{\mu \nu}
$$

Note that $g^{\mu \nu} T_{\mu \nu}=0$, with the help of the field equation for $\varphi$.

$$
D^{2} \varphi+\lambda \frac{2 d}{d-1} \varphi^{\frac{d+2}{d-2}}-\frac{d-2}{4(d-1)} R \varphi=0
$$


This ensures the vanishing of $\eta^{\mu \nu} \theta_{\mu \nu}$.

The precise form of the non-minimal coupling results in the invariance of the curved space action against Weyl transformations, involving an arbitrary function $\sigma$ [2]

$$
\begin{gathered}
g_{\mu \nu} \underset{W e y l}{\longrightarrow} e^{2 \sigma} g_{\mu \nu} \\
\varphi \underset{W e y l}{\longrightarrow} e^{\frac{2-d}{2} \sigma} \varphi
\end{gathered}
$$

The self coupling is separately invariant against (1.9). The kinetic term and the nonminimal coupling term are not, but their non-trivial response to the Weyl transformation cancels in their sum. Also it is the Weyl invariance of the action that results in the tracelessness of its $g^{\mu \nu}$-variation i.e. of $T_{\mu \nu}$, just as its diffeomorphism invariance ensures symmetry and covariant conservation of $T_{\mu \nu}$.

Thus we see that Weyl (and diffeomorphism) invariance in curved space is closely linked to conformal invariance in flat space [2]. But can a conformally invariant, flat space theory always be extended to a Weyl and diffeomorphims invariant theory in curved space? Evidently, the answer is "Yes" for the self-interacting scalar theories in $d>2$, discussed previously [3]. We now examine what happens in $d=2$.

\section{Liouville Theory: Conformally Invariant Scalar Field Dynamics in $d=2$}

A 2-dimensional model with non-trivial dynamics that is conformally invariant is the Liouville theory with Lagrange density

$$
\mathcal{L}_{0}^{\text {Liouville }}=\frac{1}{2} \eta^{\mu \nu} \partial_{\mu} \psi \partial_{\nu} \psi-\frac{m^{2}}{\beta^{2}} e^{\beta \psi}
$$

The conformal symmetry transformations act in an affine manner, so that the exponential interaction is left invariant.

$$
\delta \psi=f^{\alpha} \partial_{\alpha} \psi+\frac{1}{\beta} \partial_{\alpha} f^{\alpha}
$$

The canonical energy-momentum tensor

$$
\theta_{\mu \nu}^{\text {canonical }}=\partial_{\mu} \psi \partial_{\nu} \psi-\eta_{\mu \nu}\left(\frac{1}{2} \eta^{\alpha \beta} \partial_{\alpha} \psi \partial_{\beta} \psi-\frac{m^{2}}{\beta^{2}} e^{\beta \psi}\right)
$$

again is not traceless: $\eta_{\mu \nu} \theta_{\mu \nu}^{\text {canonical }} \neq 0$, but with an improvement it acquires that property.

$$
\theta_{\mu \nu}=\theta_{\mu \nu}^{\text {canonical }}+\frac{2}{\beta}\left(\eta_{\mu \nu} \square-\partial_{\mu} \partial_{\nu}\right) \psi, \quad \eta^{\mu \nu} \theta_{\mu \nu}=0
$$

Again $\theta_{\mu \nu}^{\text {canonical }}$ arises variationally when the Liouville Lagrange density is minimally extended by an arbitrary metric tensor. Similarly the improved tensor (2.4) is gotten when 
a non-minimal interaction is inserted.

$$
\begin{aligned}
\mathcal{L}^{\text {Liouville }}= & \frac{1}{\beta} R \psi+\frac{1}{2} g^{\mu \nu} \partial_{\mu} \psi \partial_{\nu} \psi-\frac{m^{2}}{\beta^{2}} e^{\beta \psi} \\
T_{\mu \nu}= & \frac{2}{\sqrt{|g|}} \frac{\delta}{\delta g^{\mu \nu}} \int \sqrt{|g|} \mathcal{L}^{\text {Liouville }} \\
& =\partial_{\mu} \psi \partial_{\nu} \psi-g_{\mu \nu}\left(\frac{1}{2} g^{\alpha \beta} \partial_{\alpha} \psi \partial_{\beta} \psi-\frac{m^{2}}{\beta^{2}} e^{\beta \psi}\right)+\frac{2}{\beta}\left(g_{\mu \nu} D^{2}-D_{\mu} D_{\nu}\right) \psi \\
& T_{\mu \nu} \underset{g_{\mu \nu} \rightarrow \eta_{\mu \nu}}{\longrightarrow} \theta_{\mu \nu}
\end{aligned}
$$

However, the curved-space tensor $T_{\mu \nu}$ is not traceless,

$$
g^{\mu \nu} T_{\mu \nu}=\frac{2}{\beta^{2}} R \neq 0,
$$

becoming traceless only in the flat-space limit, when $R$ vanishes. Correspondingly, the action associated with (2.5) is not invariant against Weyl transformations, which take the following form for the scalar field $\psi$.

$$
\psi \overrightarrow{\mathrm{Weyl}} \psi-\frac{2}{\beta} \sigma
$$

This formula is needed so that the interaction density $\sqrt{|g|} e^{\beta \psi}$ be invariant. However, the kinetic term together with the non-minimal term are not invariant, so that

$$
\begin{aligned}
I^{\text {Liouville }=} & \int \sqrt{|g|} \mathcal{L}^{\text {Liouville }} \\
& \underset{\text { Weyl }}{\longrightarrow} I^{\text {Liouville }}-\frac{2}{\beta^{2}} \int \sqrt{|g|}\left(R \sigma+g^{\mu \nu} \partial_{\mu} \sigma \partial_{\nu} \sigma\right)
\end{aligned}
$$

Note that the change in the action - the last term in (2.10) - is $\psi$ independent. So the field equation

$$
D^{2} \psi+\frac{m^{2}}{\beta} e^{\beta \psi}-\frac{1}{\beta} R=0
$$

enjoys Weyl symmetry, even while the action does not.

\section{Obtaining the $d=2$ Liouville theory from the $d>2$ Weyl invariant theories}

We see that the 2-dimensional situation is markedly different from what is found for $d>2$ : for the latter theories there exists a Weyl-invariant precursor, with a traceless energymomentum tensor in curved space, which leads to a traceless energy-momentum tensor in flat space. For $d=2$ the precursor is not Weyl invariant and the energy-momentum tensor becomes traceless only in the flat-space limit.

To get a better understanding of the 2-dimensional behavior, we now construct a limiting procedure that takes the Weyl invariant models at $d>2$, (1.5), to two dimensions. Thereby we expose the steps at which Weyl invariance is lost. 
In order to derive the $d=2$ Liouville theory (2.5) from the $d>2$, Weyl invariant models with polynomial interaction (1.5), we set

$$
\varphi=\frac{2 d}{\beta(d-2)} e^{\frac{\beta}{2 d}(d-2) \psi},
$$

and take the limit $d \rightarrow 2$, from above. We examine each of the three terms in (1.5) separately.

For the self interaction, we have

$$
\lambda \varphi^{\frac{2 d}{d-2}}=\lambda\left(\frac{2 d}{\beta(d-2)}\right)^{\frac{2 d}{d-2}} e^{\beta \psi} \underset{d \downarrow 2}{\frac{m^{2}}{\beta^{2}}} e^{\beta \psi} .
$$

In the last step, to absorb the singular factor we renormalize the constant $\lambda$ by defining $\frac{m^{2}}{\beta^{2}}$. For the kinetic term, the limit in immediate.

$$
\frac{1}{2} g^{\mu \nu} \partial_{\mu} \varphi \partial_{\nu} \varphi \underset{d \downarrow 2}{\longrightarrow} \frac{1}{2} g^{\mu \nu} \partial_{\mu} \psi \partial_{\nu} \psi
$$

But the non-minimal term has no limit, so we expand the exponential.

$$
\begin{aligned}
\frac{d-2}{8(d-1)} R \varphi^{2} & =\frac{d^{2}}{2 \beta^{2}(d-1)(d-2)} R e^{\frac{\beta}{d}(d-2) \psi} \\
& =\frac{d^{2}}{2 \beta^{2}(d-1)(d-2)} R+\frac{d}{2 \beta(d-1)} R \psi+\cdots
\end{aligned}
$$

In the $d=2$ limit, (3.2) and (3.3) and the last term in (3.4) lead to the curved space Liouville Lagrange density (2.5). The first term in (3.4) gives a indeterminate result in the action.

$$
\left.\int \sqrt{|g|} \mathcal{L}\right|_{d>2} \overrightarrow{d \downarrow 2} \int \sqrt{|g|} \mathcal{L}^{\text {Liouville }}+\frac{2}{\beta^{2}} \frac{\int \sqrt{|g|} R}{d-2}
$$

The indeterminancy arises from the fact that in two dimensions $\sqrt{|g|} R$ is the Euler density and its integral is just a surface term - effectively vanishing as far as bulk properties are concerned. So the last term in (3.5) gives $0 / 0$ at $d=2$. Evidently, the Liouville model is regained when $0 / 0$ is interpreted as 0 , but this leads to a loss of Weyl invariance. To maintain Weyl invariance on the limit $d \downarrow 2$, we must carefully evaluate the $\psi$-independent $\int \sqrt{|g|} R /(d-2)$ quantity - we need a kind of L'Hospital's rule for dimensional reduction.

It turns out that a precise evaluation of $\int \sqrt{|g|} R /(d-2)$ in the limit $d \downarrow 2$ can be found, by refernce to Weyl's original ideas.

Before describing this, let us remark that the conformal and Weyl transformation rules for $\psi,(2.2)$ and (2.9), are correctly obtained by substituting (3.1) into the corresponding rules for $\varphi$, (1.2) (1.9b), and passing to limit $d \downarrow 2$. The same connection exists between the equations of motion (2.11) (1.8). However, the reduction of the $\varphi$ energy-momentum tensor (1.6) produces the $\psi$ tensor (2.6) plus the term $\frac{4}{\beta^{2}} G_{\mu \nu} /(d-2)$, which is indeterminate at $d=2$, since both the numerator and denominator vanish. Notice that taking the trace of this quantity, before passing to $d \downarrow 2$, leaves $\frac{4}{\beta^{2}}(1-d / 2) R /(d-2)=-\frac{2}{\beta^{2}} R$, which cancels the non-vanishing trace of Liouville energy-momentum tensor. This again indentifies the indeterminancy as the source of Weyl non-invariance. 


\section{Weyl's Weyl Invariance}

To obtain a definite value for the behavior of $\int \sqrt{|g|} R /(d-2)$ in the limit of $d \downarrow 2$, we examine once again the Weyl transformation properties of the kinetic term for a scalar field theory in $d$ dimensions. (The self-interaction is Weyl invariant, and needs no further discussion.) As already remarked, the kinetic term is not Weyl invariant, and this is compensated by the non-minimal interaction, to produce the Weyl invariant kinetic action.

$$
I^{\mathrm{kinetic}}=\int \sqrt{|g|}\left(\frac{1}{2} g^{\mu \nu} \partial_{\mu} \varphi \partial_{\nu} \varphi+\frac{d-2}{8(d-1)} R \varphi^{2}\right)
$$

However, Weyl proposed a different mechanism for the construction of a Weyl invariant kinetic term: Rather than using a non-minimal interaction, he introduced a "gauge potential" $W_{\mu}$ to absorb the non-variance [3]. One verifies that

$$
I^{\mathrm{Weyl}}=\int \sqrt{|g|}\left(\frac{1}{2} g^{\mu \nu}\left[\partial_{\mu} \varphi+(d-2) W_{\mu} \varphi\right]\left[\partial_{\nu} \varphi+(d-2) W_{\nu} \varphi\right]\right)
$$

is invariant against (1.9), provided $W_{\mu}$ transforms as

$$
W_{\mu} \underset{\mathrm{Weyl}}{\longrightarrow} W_{\mu}-\frac{1}{2} \partial_{\mu} \sigma
$$

We now demand that $I^{\text {kinetic }}$ in (4.1) coincideds with $I^{\mathrm{Weyl}}$ in 4.2). This is achieved when the following holds.

$$
\frac{R}{4(d-1)}=D^{\mu} W_{\mu}+(d-2) g^{\mu \nu} W_{\mu} W_{\nu}
$$

this curious Riccati-type equation is familiar in $d=2$, where it states that $\sqrt{|g|} R$ is a total derivative; a condition that is generalized by (4.4) to arbitrary $d>2$.

With the help of (4.4) we evaluate, before passing to $d \downarrow 2$, the ambiguous contribution to the action - the last term in (3.5). We have from (4.4)

$$
\frac{\int \sqrt{|g|} R}{4(d-1)(d-2)}=\frac{1}{d-2} \int \partial_{\mu}\left(\sqrt{|g|} W^{\mu}\right)+\int \sqrt{|g|} g^{\mu \nu} W_{\mu} W_{\nu} .
$$

The first term does not contribute, even when $d \neq 2$, because the integrand is a total derivative for all $d$, while the remainder leaves

$$
\lim _{d \downarrow 2} \frac{\int \sqrt{|g|} R}{d-2}=4 \int \sqrt{|g|} g^{\mu \nu} w_{\mu} w_{\nu}
$$

where $\left.w_{\mu} \equiv W_{\mu}\right|_{d=2}$ satisfies, according to (3.4),

$$
4 D^{\mu} w_{\mu}=R \quad \text { at } d=2 .
$$

[Note that (4.3) and (4.7) are consistent with the Weyl transformation formula for $R$ at

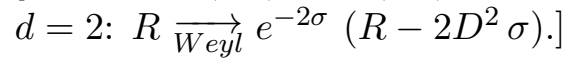


Thus to achieve Weyl invariance, the action should be supplemented by the metricdependent, but $\psi$-independent term.

$$
\triangle I=\frac{8}{\beta^{2}} \int \sqrt{|g|} g^{\mu \nu} w_{\mu} w_{\nu}
$$

According to (4.3) and (4.7), the Weyl variation of $\triangle I$ is

$$
\triangle I \underset{W e y l}{\longrightarrow} \triangle I+\frac{2}{\beta^{2}} \int \sqrt{|g|}\left(R \sigma+g^{\mu \nu} \partial_{\mu} \sigma \partial_{\nu} \sigma\right) .
$$

This cancels the Weyl non-invariant response of $I^{\text {Liouville; see (2.10). }}$.

It remains to determine $w_{\mu}$ by solving (4.7). We are of course interested in a local solution, so that the Weyl-invariant Liouville action be local. Such a solution has been found [4. It is

$$
w^{\mu}=\frac{\varepsilon^{\mu \nu}}{4 \sqrt{|g|}}\left(\frac{\varepsilon^{\alpha \beta}}{\sqrt{|g|}} \partial_{\alpha} g_{\beta \nu}+(\cosh \omega-1) \partial_{\nu} \gamma\right) .
$$

The second term in the parenthesis is the canonical SL $(2, \mathrm{R})$ 1-form, with

$$
\cosh \omega=\frac{g_{+-}}{\sqrt{|g|}} \text { and } e^{\gamma}=\sqrt{\frac{g_{++}}{g_{--}}} .
$$

$\left[(+,-)\right.$ refer to light-cone components $\left.\frac{1}{\sqrt{2}}\left(x^{0} \pm x^{1}\right).\right]$ This portion of $w^{\mu}$ is Weyl invariant, while the rest verifies the transformation law (4.3). The solution (4.10) is not unique. One may add to (4.10) any Weyl-invariant term of the form $\frac{\varepsilon^{\mu \nu}}{\sqrt{|g|}} \partial_{\nu} X$, since this will not contribute to (4.7).

Remarkably $w^{\mu}$ in (4.10) is not a contravariant vector, even though $D_{\mu} w^{\mu}$ is the scalar $R / 4$. Consequently our Weyl invariant Liouville action $I^{\text {Liouville }}+\triangle I$ is not diffeomorphism invariant. Its $g^{\mu \nu}$ variation defines a traceless energy-momentuim tensor, which however is not (covariantly) conserved.

We do not know what to make of this. Perhaps the above mentioned ambiguity can be used to remedy the diffeomorphism non-invariance, but we have not been able to do so. It would seem therefore that a local, curved-space Liouville action can be either diffeormorphism invariant or Weyl invariant, but not both.

If this conjecture is true, we are facing an "anomalous" situation in a classical field theory, which has previously been seen only in a quantized field theory. It is know that in two dimensions, the diffeomorphism invariant Lagrange density $\frac{1}{2} \sqrt{|g|} g^{\mu \nu} \partial_{\mu} \varphi \partial_{\nu} \varphi$ is also invariant against Weyl transformations that transform the metric tensor, but not the scalar field $\varphi$ [i.e. Eq. (1.9) at $d=2$ ]. However, the effective quantum action that is obtained by performing the functional integral over $\varphi$, yields a metric expression which is either diffeomorphism invariant or Weyl invariant but not both [5].

If locality is abandoned, one may readily construct a covariant solution for $w_{\mu}$ in the form $\partial_{\mu} w$

$$
\omega_{\mu}=\partial_{\mu} \omega
$$

with $w$ transforming under a Weyl transformation as [compare (4.3)]

$$
w \underset{\text { Weyl }}{\longrightarrow} w-\frac{\sigma}{2} \text {. }
$$


Evidently

$$
\begin{gathered}
D^{2} w=R / 4, \\
w(x)=\frac{1}{4} \int d^{2} y \sqrt{|g(y)|} \frac{1}{D^{2}(x, y)} R(y),
\end{gathered}
$$

where the Green's function is defined by

$$
D_{x}^{2} \frac{1}{D^{2}(x, y)}=\frac{1}{\sqrt{|g|}} \delta^{2}(x-y) .
$$

Eq. (4.13) is verified by (4.14), and the addition to the Liouville action is just the Polyakov action [5].

$$
\triangle I=\frac{1}{2 \beta^{2}} \int \partial^{2} x d^{2} y \sqrt{|g(x)|} R(x) \frac{1}{D^{2}(x, y)} \sqrt{|g(y)|} R(y)
$$

This then provides a diffeomorphism and Weyl invariant action for the Liouville theory, which however is non-local. Whether locality can be also achieved remains an open question.

\section{References}

[1] C. Callan, S. Coleman and R. Jackiw, "A New Improved Energy Momentum Tensor," Ann. Phys. 59, 42 (1970).

[2] See e.g. B. Zumino, "Effective Lagrangians and Broken Symmetries," in Lectures on Elementary Particles and Quantum Field Theory, S. Deser, M. Grisaru and H. Pendleton, eds. (MIT, Cambridge MA 1970).

[3] See e.g. A. Iorio, L. O'Raifeartaigh, I. Sachs and C. Wiesendanger, "Weyl Gauging and Conformal Invariance," Nucl. Phys. B495, 433 (1997).

[4] S. Deser and R. Jackiw, "Energy-Momentum Tensor Improvements in Two Dimensions," IJMP B 10, 1499 (1996).

[5] A. Polyakov, Gauge Fields and Strings, (Harwood, Chur, Switzerland, 1987). 